zelne Bacillen mit central gelegenen Sporen?. A3 Gruppen von Einzelbacillen. A4 Bacillus mit endständiger und centraler Spore?. Oelimmersion Hartnack Ocul. 4. A5 Bacillenketten, A6 Bacillenbaufen System 8 Ocul. 3 Hartnack. B1 Kette vou kurzen Bacillen mit endständigen Plasmaklumpen. B2 Einzelne kurze Bacillen mit endständigen Plasmaklumpen, B2' eben solche nach dem Mikroskopbild vergrössert. B3 Kurze Bacillen mit in Auflösung begriffener Hülle, B3' eben solche nach dem Mikroskopbild vergrössert. B4 Häufchen kurzer Bacillen Oelimmersion Hartnack Ocul. 4.

\title{
XXIV.
}

\section{Ein Fall von Myoma striocellulare am Hoden.}

\author{
Von Prof. E. Neumann in Königsberg.
}

(Hierzu Taf. XI. Fig. 1-2.)

Im Jahre 1849 beschrieb Rokitansky ${ }^{1}$ ) eine aus quergestreiften Muskelfasern bestehende Hodengeschwulst, welche als erstes sicher constatirtes Beispiel des Vorkommens solcher Geschwülste von besonderem Interesse ist. Der Tumor hatte sich bei einem 18jährigen Manne, angeblich in Laufe von 4 Monaten, bis zur Grösse eines Gänseeies entwickelt und erschien, ohne den Hoden selbst zu betheiligen, in die Tunica albaginea desselben eingewebt, seine Substanz war zum grössten Theile blass, prallelastisch, fleischig, die seinen Hauptbestandtheil bildenden Muskelfasern ähnelten denen des Herzens oder, wie Rokitansky bei anderer (Gelegenheit ${ }^{2}$ ) sagt, denen des embryonalen Herzens. Die musculöse Beschaffenheit der Geschwulst ist später auch durch eine von Virchow ${ }^{3}$ ) and $\mathrm{Heschl}^{4}$ ) vorgenommene Nachuntersuchung bestätigt werden.

Obwohl nun seitdem eine grössere Zahl von Benbachtungen über das Myoma striocellulare in der Literatur sich angesammelt hat, so steht doch der obige Fall Rokitansky's meines Wissens

1) Rokitansky, Zeitschrift der Gesellsch. d. Aerzte zu Wien. Jahrg. V.

$\Rightarrow$ Lehrbuch d. patholog. Anatomie. 2. Aufl. I. S. 190.

3) Virchow, Würzburger Verhandlungen. T. S. 188.

4) Heschl, Dieses Archiv Bd. 8. S. 126. 
in Bezug auf den Sitz und die Beschaffenheit der Geschwulst noch immer als ein Unicum dar, allerdings sah man noch einige Male ${ }^{1}$ ) quergestreifte Muskelfasern in teratoiden Hodengeschw ülsten auftreten, immer jedoch combinirt mit mannichfachen anderen, die Hauptmasse der Geschwulst bildenden Bestandtheilen und einen Befund von untergeordneter Bedeutung darstellend. Um so mittheilenswerther dürfte die folgende Beobachtung sein, welche unzweifelhaft eine sehr nahe Verwandtschaft mit Rokitansky's Falle hat, so dass man wohl einen übereinstimmenden Ursprung vermuthen darf.

Das betreffende Präparat wurde am 25. September d. J. dem Pathologischen Institut von Herrn Collegen Professor Schneider übergeben und besteht aus dem durch Castration entfernten linken Hoden eines $3 \frac{1}{2}$ jährigen Knaben, bei welchem erst wenige Monate zuvor (in der zweiten Hälfte des Juli) die ersten Spuren einer Geschwulstbildung von den Eltern wahrgenommen worden waren, so dass jedenfalls die Cebereinstimmung mit der Angabe von Rokitansky, ein auffallend schnelles Wachsthum seitdem stattgefunden haben musste. Wie die anatomische Untersuchung ergiebt, ist sowohl der Hoden selbst (Fig. $1 \mathrm{H}$ ) als der Nebenhoden (N) in völlig normalem Zustande vorhanden, dagegen haftet dem unteren Pole des ersteren ein eiförmig abgerundeter Tumor ( $\mathrm{T}, \mathrm{T}^{\prime}$ ) von etwa Wallnussgrösse an. Aeusserlich erscheinen Hoden und Geschwulst fest miteinander verschmolzen and nur durch eine seichte Furche geschieden, auf dem Durchschnitte sieht man jedoch die einen ununterbrochenen Ueberzug um die Hodensubstanz bildende Albuginea als scharfe Grenze gegen das Tumorgewebe. Die Tunica vaginalis propria (Pr) in der Zeichnung von der lateralen Seite her eröffnet und zurückgeschlagen, setzt sich vom Hoden aus auf die Geschwulstoberfläche eine Strecke weit fort, so dass ein 'Theil (T)'des Tumor in den Sack der Scheidenhaut eingestülpt, ein anderer $\left(\mathrm{T}^{\prime}\right)$ extravaginal und nur von der allgemeinen Scheidenhaut umhült erscheint. Der Nebenhode ist mit seinem gleichfalls ausserhalb der Vaginalis propria gelegenen Schweife mittelst lockeren Zellgewebes mit der Geschwulst verbunden, hat aber keine näheren Beziehungen zu derselben.

1) Billroth, ibid. VIII. S. 433 ; Senftleben, ibid. XV. S. 345. 
Was die Beschaflenheit der Neubildung betrifft, so hat dieselbe eine gleichmässig feste, prallelastische Consistenz, die Schnittfläche ist blass, grauröthlich, feuchtglänzeud, leicht vorquellend; bei dem Versuche, das Gewebe zu zerzupfen, erweist es sich als äusserst zäh, cohärent, von fasriger Structur. Konnte hiernach die Vermuthung entstehen, dass es sich um ein Fibrom oder ein derbes Fibrosarcom handle, so gelingt es der mikroskopischen Untersuchung leicht, das Eigenartige des histologischen Baues aufzudecken. In ein ziemlich spärliches, festgefügtes, fibrilläres Bindegewebe eingelagert, zeigen sich als dominirende Elemente, musculöse Gebilde, von verschiedenster Grösse und Form (Fig. 2). Neben gut ausgebildeten, eines Sarcolemms freilich scheinbar entbehrenden, kernreichen, quergestreiften Muskelfasern, welche bei durchschnittlicher Breite von 10 bis $15 \mu$ öfters in einer Längenausdehnung von 200 bis $300 \mu$ zu verfolgen waren, fanden sich andere Fasern, welche durch eine ausgeprägt varicsöe Form ausgezeichnet waren, so dass in ihnen mächtige kernhaltige Aufschwellungen mit ganz schmalen Verbindungsstücken wechselten, wie bei gewissen Formen der Muskelatrophie; ferner gab es zahlreiche Fasern, welche gauz der embryonalen Entwicklungsform der Muskelfasern entsprechen und sich als grosse Spindelzellen darstellten mit quergestreiftem Randsaum und centraler, die Kerne umschliessende Protoplasmasubstanz. Auffallender ist der Befund von kugligen oder unregelmässig scholligen, zelligen Gebilden (Fig. 2 b, b, b), welche fast überall in grösserer oder geringerer Zahl zwischen den beschriebenen fasrigen Elementen eingelagert sind und die an Schnitten leicht als Querschnittsbilder derselben gedeutet werden konnten, was jedoch durch Zerzupfungspräparate widerlegt wird. Diese Gebilde sind bei einem Durchmesser von 10 bis $30 \mu$ je nach ihrer Grösse bald nur mit einer einfachen bald mit mehreren, in Gruppen zusammengelagerten Kernen versehen, welche, wie die Kerne der Muskelfasem, ein bläschenähnliches Aussehen haben und meist deutliche Nucleolen erkennen lassen; eine Querstreifung fehlt ihnen natürlich, wohl aber erinnern sie häufig durch den starken Glanz und ein etwas zerklüftetes Aussehen ihrer Substanz an Muskelfasern im Zustande der sogenannten "wachsartigen Degeneration"; einige Male schien sich eine zarte Sarcolemma- ähnliche Hülle von ihrer 
Peripherie abzuheben. An ihrer musculösen Natur konnte um so weniger ein Zweifel sein, als sie sowohl in ihrer Form alle Uebergänge zu langgestreckten Bändern und wirklichen Muskelfasern darboten als auch in ihrer chemischen Reaction mit diesen völlig übereinzustimmen schienen. Der Behandlang mit 33procentiger Kalilauge oder 20procentiger Salpetersäure leisteten sie denselben Widerstand wie die Muskelfasern, und liessen sich durch dieselbe in grosser Zahl isoliren, womit zugleich der Beweis geführt war, dass sie eine selbständige Existenz besassen und nicht etwa durch Zerzupfen künstlich abgelöste Theile längerer Fasern darstellten. Pikrocarminborax - Salzsäure färbte sie, wie die Muskelfasern gelb, Hämatoxylin bläulichgrau, Osmium schwärzlichgrau, Goldchlorid violett. Auch an Schnitten, welche nach der Erhärtung in den verschiedensten Flüssigkeiten (Alkohol, Chromsäure, Müller'sche Flüssigkeit, Osmium) angefertigt wurden, liessen sie sich überall scharf von den, kleine schmale Spindelfiguren darstellenden Bindegewebszellen unterscheiden.

Es konnte sich bei der Deutung dieser Gebilde demnach nur um die Frage handeln, ob sie einfach als in der Entwickelung zurückgebliebene Vorstufen der in der Geschwulst vorhandenen ausgebildeten Muskelfasern oder vielmehr als in fehlerhafter Richtung entwickelte, missbildete, gewissermaassen krüppelhafte Muskelfasern zu betrachten seien? Wenn ich mich im Einklange mit Marchand, welcher kürzlich in einem von ihm beobachteten und genau beschriebenen interessanten Falle am Myosarcom ganz ähnliche "kuglige Muskelfasern " fand'), für die letztere Auffassung entscheide, so darf ich mich wohl darauf berufen, dass die beschriebenen Formationen den bekannten normalen Entwicklungsstufen quergestreifter Muskelfasern durchaus fremd sind und dass die Dimensionen vieler derselben den Durchmesser der gleichzeitig vorhandenen Muskelfasern bedeutend überschritten, denn während letztere eine Maximalbreite von etwa $20 \mu$ besassen, erreichten jene kugligen und scholligen Gebilde mit einem Durchmesser von $30 \mu$ öfters die Grösse ansehnlicher Myeloplaques. Ich kann hierbei nicht umhin, die Frage aufzuwerfen, ob nicht vielleicht auch manche von früheren Untersuchern derartiger Geschwülste erwähnten und aus dem Bindegewebe

1) Marchand, Dieses Archiv Bd. C. S. 42. 
abgeleiteten, demnach als Sarcomzellen beschriebenen Rundzellen in Wirklichkeit die Bedeutung musculöser Elemente hatten, jedenfalls passte die Bezeichnung Myosarcom für unseren Fall nicht, da die Zellen des Bindegewebsstromas spärlich und wenig entwickelt waren.

Es sei noch hinzugefügt, dass in sämmtlichen beschriebenen musculösen Gebilden, in den nach normalem Typus entwickelten sowohl wie in den atypischen Formationen häufig zahlreiche, in Osmium sich schwärzende Fetttröpfchen als Zeichen einer beginnenden regressiven Metamorphose angehäuft waren. Dagegen ergab die Untersuchung auf einen Glycogengehalt derselben, zu welchem die bemerkenswerthen Beobachtungen Marchand's (l. c.) in seinem Falle aufforderten, ein negetives Resultat, womit auch die nur wenig succulente Beschaffenheit des Tumors und der Mangel einer durchtränkenden schleimig visciden Flüssigkeit übereinstimmte.

Ueber den Ursprung der Geschwulst lassen sich natürlich nur Vermuthungen äussern. Das jugendliche Alter des übrigens ganz gesunden kleinen Patienten legt gewiss die Annahme einer congenitalen Anlage nahe, indessen dürfte kaum eine Nöthigung dafür vorliegen, auf in Folge abnormer embryonaler Entwicklungsvorgänge aberrirte und transplantirte "Muskelkeime" zu recurriren. Wie aus Beschreibung und Abbildung ersichtlich sein wird, entspricht nehmlich der Sitz der Geschwulst genau einem embryonalen Gebilde musculöser Natur, dem Gabernaculum Hunteri und eine in früher Zeit angelegte, später zu weiterer und zwar ziemlich rapider Ausbildung gelangte Proliferation seiner musculösen Bestandtheile würde die Geschichte des Tumor füglich zu erklären im Stande sein. Bestimmte weitere Anhaltspunkte für diese Annahme vermag ich allerdings nicht anzuführen; zur Beurtheilung des Werthes derselben erschien es mir jedoch von Wichtigkeit, zu wissen, ob es auch in dem oben erwähnten Rokitansky'schen Falle, in welchem durch die Beschreibung des berühmten Autors der Sitz der Geschwulst nur sehr unbestimmt bezeichnet worden ist, zulässig ist, an eine Beziehung derselben zu dem Gubernaculum Hunteri zu denken, eine Vermuthung, welche, wie ich finde, bereits vor langer Zeit Kölliker (Mikroskopische Anatomie I, S. 259) ausgesprochen hat. Wenn sich schon zu 
Gunsten derselben anführen lässt, dass Rokitansky in seiner Patholog. Anatomie (l. c.) Kölliker's Hypothese erwähnt, ohne sie zurückzuweisen, was er vermuthlich nicht unterlassen hätte, wenn die anatomischen Verhältnisse der Geschwulst ihr bestimmt widersprächen, so genügte mir das doch nicht und ich wandte mich daher an Herrn Kollegen Kundrat in Wien mit der Bitte um nähere Auskunft über das, wie ich voraussetzen durfte, in der pathologisch-anatomischen Sammlung daselbst aufbewahrte Präparat. In dem Bericht, den derselbe mir hierauf freundlichst erstattete, heisst es, "Hoden und Tumor sind in dem grössten Durchmesser der Längsrichtung nach durchtrennt; von der so hergestellten Schnittfläche besonders, aber auch von einzelnen Stellen der Peripherie der Geschwulst sind kleine Stücke ausgeschnitten; immerhin lässt sich deutlich erkennen, dass auch diese Geschwulst grossentheils extravaginal gelagert war, denn der grössere untere Antheil ist nur von verdichteten Bindegewebslagen, welchen noch Gewebe der Dartos anhaftet, überkleidet und nur im oberen Theile lässt sich die Tunica vaginalis propria nachweisen. Am Durchschnitte sind die Verhältnisse beiliegender Skizze (s. d. Holzschnitt) entsprechend, an der seitlichen Peripherie reicht die Tun. vag. pr. etwas weiter auf die Geschwulst herab."

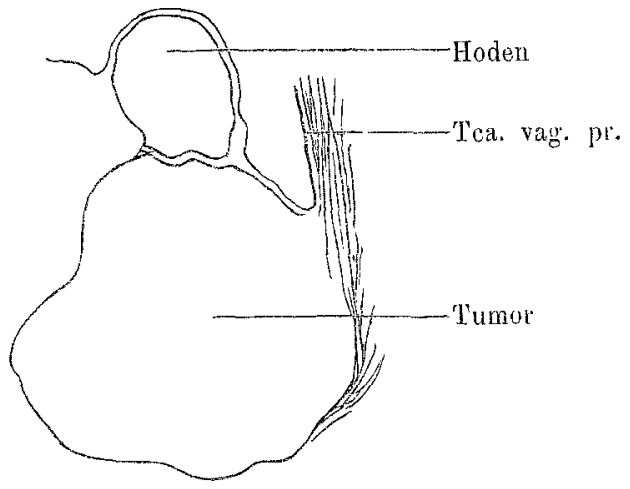

Nach diesen Angaben Kundrat's kann es wohl kaum zweifelhaft sein, dass auch in dem Rokitansky'schen Falle die Geschwulst an demjenigen Theile der Hodenperipherie ihren Sitz hatte, welcher der Anhaftungsstelle des embryonalen Gubernacu- 
lum Hunteri entspricht, nehmlich an der Umschlagsstelle der Tunica vaginalis propria am unteren Hodenpole, und es würde somit in der That auch hier dieses Gebilde als Ausgangspunkt für die Entwicklung der Neubildung in Betracht kommen.

\title{
Erklärung der Abbildungen. Iafel XI.
}

Fig. 1. H Hoden. N Nebenhoden (Caput und Corpus). TT' Tumor. Pr T'unica propria testis (eröffnet und zurückgeschlagen). F sp Funiculus spermaticus.

Fig. 2. a a Muskelfasern. b b b Kuglige Muskelelemente. c c Bindegewebszellen. d Fibrilläre Substanz.

\section{XXV.}

\section{Ueber eimen Fall von Entwicklungshemmung} des Herzens.

\author{
(Aus der medicinischen Poliklinik zu Berlin.) \\ Von Dr. med. et phil. Hans Leo, \\ Assistenten an der medic. Universitäts-Poliklinik zu Berlin.
}

(Hierzu Taf. XI. Fig. A u. B.)

Die Zahl der am Herzen vorkommenden Entwicklungsfehler ist eine so grosse, dass fast jede nur denkbare Anomalie in der That schon zur Beobachtung gelangt ist. Der vorliegende Fall beansprucht, abgesehen von dem interessanten anatomischen Befunde, auch deshalb besonderes Interesse, weil derselbe längere Zeit intra vitam beobachtet werden konnte und die in so bedeutendem Maasse am Herzen vorhandenen Abnormitäten sich bei häufig wiederholter Untersuchung nur in verhältnissmässig geringer Weise documentirten.

Atresie der Arteria pulmonalis. Rudimentäre Entwicklung des rechten Ventrikels. Offenbleiben des Ductus Botalli. Offenes Foramen ovale. Abnorm entwickelte Membranen im rechien Vorhofsraum.

Adele Wehner wurde am 29. Dec. 1884 als Kind armer, aber gesunder Eitern geboren. Auch die übrigen vier Kinder der Eltern sind gesund. 

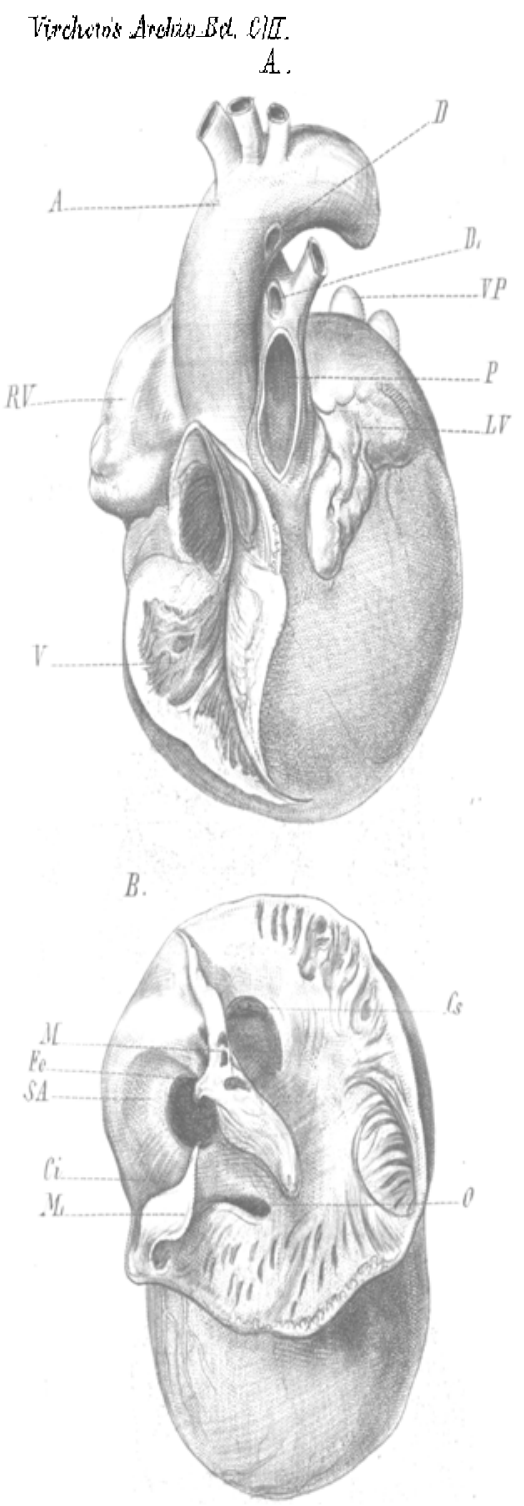

Lipyritu dus

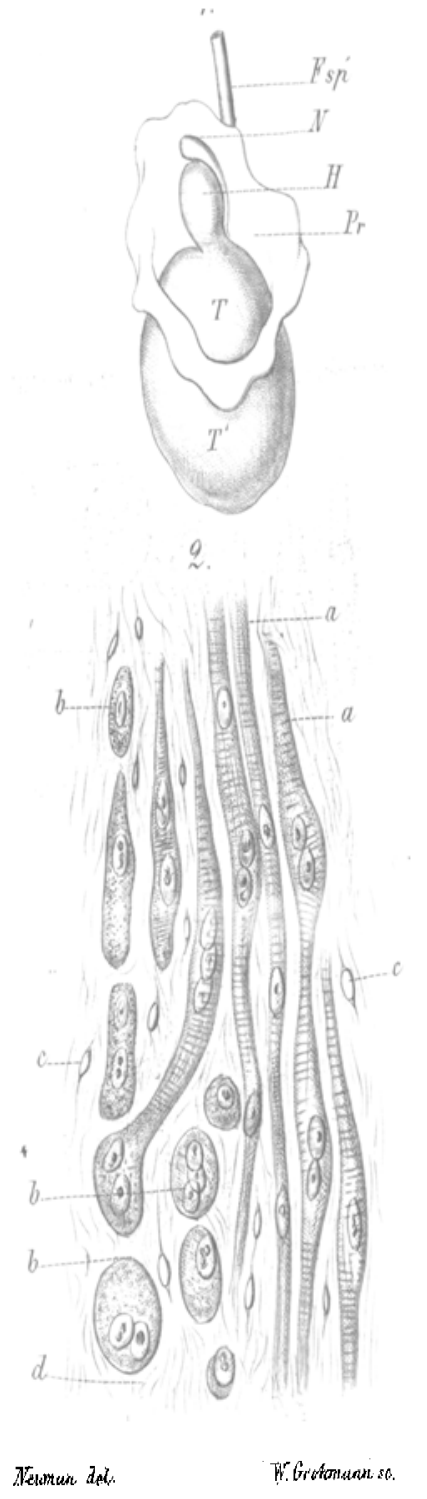

